\title{
EXPERIMENTAL MODAL ANALYSIS OF A CANTILEVER BEAM USING A WIRELESS SENSOR NODE FOR STRUCTURAL HEALTH MONITORING
}

Surgwon Sohn ${ }^{1}$

Abstract- Structural health monitoring (SHM) is crucial for protecting structures against the wind, aging, and potential collapse. Modern wireless-based SHM has two main constraints: cost and power consumption. Micro electro-mechanical system (MEMS) accelerometers meet these conditions. A wireless sensor node is also important since it deals with vibration data acquisition and wireless transmission. This paper presents an experimental modal analysis of a cantilevered steel beam using a low-cost wireless sensor node for SHM in order to demonstrate the feasibility of its use. We use a commercial, off-theshelf wireless sensor node, Waspmote, as a data acquisition and transmission board. Waspmote has a built-in MEMS accelerometer, LIS331DLH, and a daughter board for IEEE 802.15.4 wireless transmission protocol. Dynamic characteristics of the structures, such as natural frequencies, damping ratios, and mode shapes are acquired. These dynamic properties are here compared with the results of a conventional wired vibration measurement system.

Keywords - Experimental Modal Analysis, Cantilever Beam, Wireless Sensor Node, Structural Health Monitoring

\section{INTRODUCTION}

Structural health monitoring (SHM) is crucial for protecting structures against wind, aging, and potential collapse. Since wireless sensor nodes reduce the time and cost of installation and maintenance for the SHM, the use of wireless sensor networks (WSNs) has recently become an area of interest [1]. Modern wireless-based SHM has two main constraints: cost and power consumption. Micro electro-mechanical system (MEMS) accelerometers meet these conditions. A wireless sensor node is also important since it deals with vibration data acquisition and wireless transmission. We choose a commercial low-cost wireless sensor node that is composed of a MEMS accelerometer, Arduino compatible processor board, and IEEE 802.15.4 communication module.

To better understand any structural dynamic problems, the resonant frequencies of a structure need to be identified and quantified. Today, experimental modal analysis has become a widespread means of finding the modes of vibration of a structure. Modal analysis is the field of measuring and analyzing the dynamic response of structures during excitation [2]. This paper presents an experimental modal analysis of a cantilevered steel beam using a low-cost wireless sensor node for SHM in order to demonstrate the feasibility of its use. Dynamic characteristics of the structures, such as natural frequencies, damping ratios, and mode shapes are acquired. These dynamic properties are here compared with the results of a conventional wired vibration measurement system.

\section{THEORETICAL ANALYSIS OF A CANTILEVER BEAM}

A beam is said to be a cantilever beam if one end is fixed to a support and the other end is free to move. Consider a thin uniform cantilever beam with length $\mathrm{L}$ as shown in Figure 1, where E is the elastic modulus (aka Young's modulus), I is the moment of inertia, $f(x)$ is displacement in $y$ direction at distance $x$ from fixed end, $\omega_{n}$ is the circular natural frequency, $m$ is the mass per unit length. A closed form solution of the circular natural frequency, $\omega_{n}$, can be written as

$$
\omega_{n}=\alpha_{n}^{2} \sqrt{\frac{E I}{m L^{4}}}
$$

where $\alpha_{1}=1.875, \alpha_{2}=4.694, \alpha_{3}=7.855$. Thus, first three mode shapes $\left(f_{1}(x), f_{2}(x), f_{3}(x)\right)$ and natural frequencies $\left(\omega_{1}, \omega_{2}\right.$, $\left.\omega_{3}\right)$ are shown in Figure 1.

Now, if we consider the mass of accelerometer, $m_{a c c}$ at the free end of the beam, then the total mass at free end will be

$$
M=m_{e f f}+m_{a c c}
$$

So for the discrete beam with accelerometer, the theoretical first natural frequency after considering the mass of accelerometer will be

$$
\omega_{1}=\sqrt{\frac{K}{M}} \quad \text { with } \quad K=\frac{3 E I}{L^{3}}
$$

\footnotetext{
${ }^{1}$ Dept. Of Computer and Information Eng., Hoseo University, Chungnam 31499, Rep. of Korea
} 
Now, let us consider that two accelerometers are used. The mass of the first wire-based accelerometer would be 51 gram, and in this case, coaxial cable must be considered, too. From the Equation (3), we get the first circular natural frequency as follows.

$$
\omega_{1-a c c 1}=\sqrt{\frac{3 \times 2.1 \times 10^{11} \times 1.35 \times 10^{-10}}{(0.309+0.051) \times 0.9^{3}}}=18.00(\mathrm{rad} / \mathrm{sec})
$$

So, $f_{1 \_a c c 1}=\omega_{1 \_a c c l} / 2 \pi=2.87 \mathrm{~Hz}$. If we consider the weight of coaxial cable, then the frequency would be lower than this value.

In the second case, the type of accelerometer is Waspmote embedded wireless accelerometer, so it is not necessary to consider the cable weight, but we have to consider the battery weight instead. The total weight would be 175 gram. Then

$$
\omega_{1 \_ \text {acc } 2}=\sqrt{\frac{3 \times 2.1 \times 10^{11} \times 1.35 \times 10^{-10}}{(0.309+0.175) \times 0.9^{3}}}=15.53(\mathrm{rad} / \mathrm{sec})
$$

So, $f_{1 \_a c c 2}=\omega_{1 \_a c c 2} / 2 \pi=2.47 \mathrm{~Hz}$.

In our case, we use PCB 603C01 ICP type accelerometer which has a weight of 51gram. In other case, we use also LIS331DLH MEMS type accelerometer embedded into a Waspmote sensor node which has a weight of 20gram. In addition to the Waspmote, Lithium ion battery of $6600 \mathrm{~mA}$ with $155 \mathrm{gram}$ is attached to the Waspmote, so total weight of wireless accelerometer becomes 175 gram.

\section{EXPERIMENTAL MODAL ANALYSIS OF A CANTILEVER BEAM}

MEMS accelerometers and wireless sensor nodes provide advantages of low-cost and low power consumption. These two modules are combined to deal with vibration data acquisition and wireless data transmission to the remote host computer. To replace the costly, conventional wired IEPE (Integrated Electronics Piezo Electric) or ICP (Integrated Circuit Piezoelectric) accelerometer-based vibration monitoring system, we need to justify the performance of the wireless vibration measurement system.

Functional subsystems of WSN are sensing interface, computational core, and actuation interface as shown in the previous research [3]. By keeping in mind the low-resources, we have selected a commercial off-the-shelf product, Libelium Waspmote [4], that includes ATmega 1281 micro-controller. It has $8 \mathrm{~Kb}$ SRAM, $4 \mathrm{~Kb}$ EEPROM, $128 \mathrm{~Kb}$ flash memory, and 2 Gb SD memory. For saving the power consumption, there are 4 modes; On: $15 \mathrm{~mA}$, Sleep: $55 \mu \mathrm{A}$, Deep sleep: $55 \mu \mathrm{A}$, Hibernate: 0.7 $\mu \mathrm{A}$.

A low cost accelerometer sensor, LIS331DLH, is built into the Waspmote that is used to measure the accelerations of the cantilever beam. It has three scales; $\pm 2 \mathrm{~g}, \pm 4 \mathrm{~g}, \pm 8 \mathrm{~g}$, and seven work modes which is important in case of battery operated products. In normal mode, the output data rate can be one of $50 \mathrm{~Hz}, 100 \mathrm{~Hz}, 400 \mathrm{~Hz}$, or $1000 \mathrm{~Hz}$. But in low power mode, it can be one of $0.5 \mathrm{~Hz}, 1 \mathrm{~Hz}, 2 \mathrm{~Hz}, 5 \mathrm{~Hz}$, or $10 \mathrm{~Hz}$. A ZigBee-802.15.4-Pro 802.15.4 rf module is used for a wireless data transmission to satisfy the low resources. It operates at $-100 \mathrm{~dB}$ sensitivity within the ISM $2.4 \mathrm{GHz}$ frequency band. To interface this rf module on the Waspmote, it needs a Waspmote gateway. It communicates with the host PC via IEEE 802.15.4/ZigBee protocol. To investigate a suitability of low-resource wireless sensor node in a SHM, an experimental modal analysis for a cantilevered steel beam is carried out. The frequency response function was performed at the host PC using Smart Office Analyzer software [5] with the 1,024 sample of data.

To study the accuracy of the dynamic acceleration measurement obtained with a wireless accelerometer such as LIS331DLH on the Waspmote, we used a conventional wired IEPE accelerometer to measure the transient response of a cantilever beam simultaneously. The experimental modal analysis of a simple cantilever beam was performed to demonstrate the use of wireless sensor nodes.

A cantilevered steel beam was used for the experiment with approximate dimensions of $900 \times 60 \times 3 \mathrm{~mm}$. An IEPE or ICP type impact hammer, model $086 \mathrm{C} 03$ of PCB Piezotronics, was used as the excitation source at any point input and response data was acquired at the point of the beam. A cable with the hammer is connected to the NI-9234 as data acquisition hardware. A wired accelerometer, 603C01 of PCB Piezotronics, was used for the reference. A MEMS accelerometer, LIS331DLH built on the Waspmote, is located at the end of the cantilever beam and the excitation was imparted on the point as shown in Figure 1. 


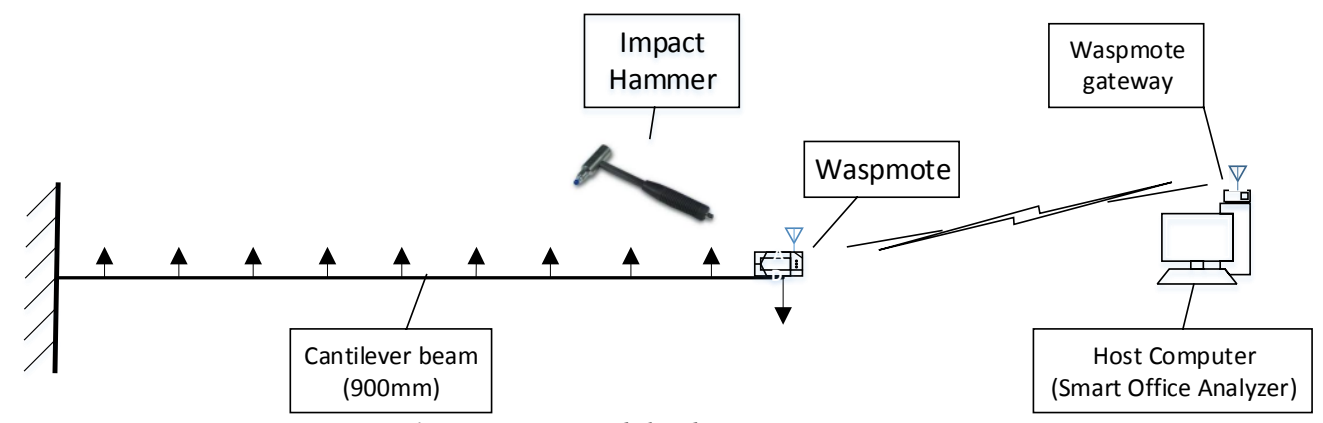

Figure 1. Test setup and the data acquisition points

In this test, we use a fixed accelerometer and a roving hammer as excitation and give a multiple-input, single-output (MISO) analysis. We have chosen 10 excitation points as an input force to obtain 3 mode shapes. A frequency response function is acquired by computation of the cross power spectrum of the input and output vibration data using commercial Smart Office Analyzer software. The measurement for experimental modal analysis is to acquire frequency response function data from a test structure. As the cantilever beam setup was excited with the hammer manually the response of the structure was measured in the form of acceleration. Both the excitation and response signal were sent to the data acquisition hardware with a signal processing software. The Waspmote communicates with the host PC using a gateway device via the IEEE 802.15.4 protocol.

Sampling rate of the wired ICP accelerometer was $256 \mathrm{~Hz}$, and the vibration signal was collected for 4 seconds, so a total of 1,024 samples were recorded for post-processing. In case of wireless accelerometer, the sampling rate of the LIS331DLH was $400 \mathrm{~Hz}$, but the vibration data was recorded at a rate of $256 \mathrm{~Hz}$ for 4 seconds.

\section{PERFORMANCE EVALUATION OF WIRELESS ACCELEROMETERS}

Ten excitation points are chosen and three mode shapes are obtained from the commercial Smart Office Analyzer software. These mode shapes are represented in Fig. 2. An FRF [6] is acquired by computation of the cross power spectrum of the input and output vibration data. The modal frequencies were estimated as the frequencies where we received maximum gain in the FRF plots [7]. While Fig. 3 depicts the FRF in case of wired ICP accelerometer, Fig. 4 represents the FRF of the wireless accelerometer. Both cases produced the same natural frequencies as in Fig. 3 and Fig. 4.

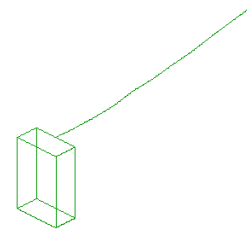

(a) $1 \mathrm{st}$ mode

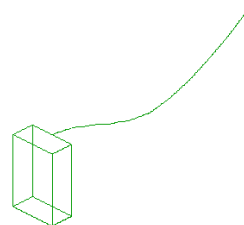

(b) 2nd mode

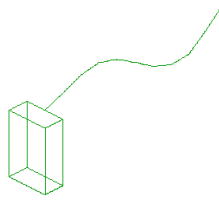

(c) 3rd mode

Figure 2. Three mode shapes of a cantilevered steel beam

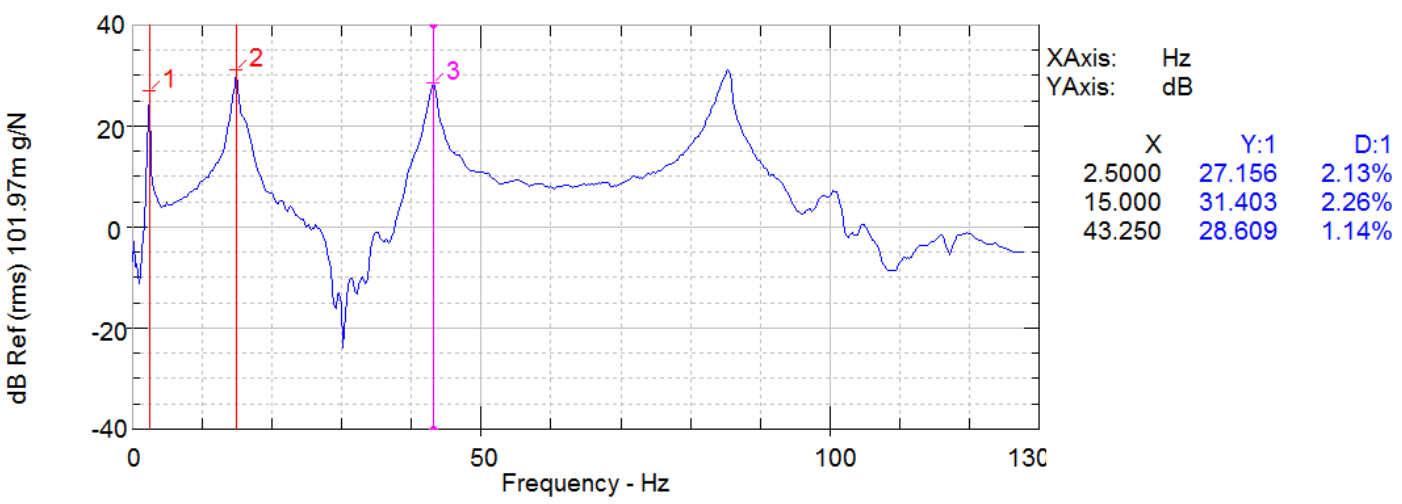

Figure 3. Natural frequencies and damping ratios of a cantilever beam using a wired ICP accelerometer (PCB 603C01). 


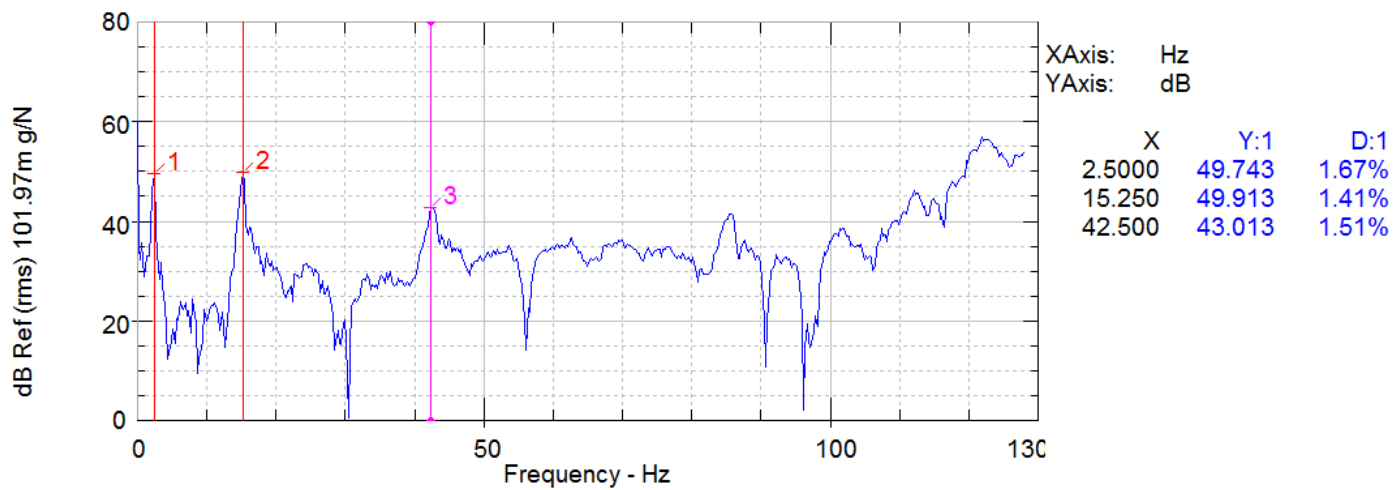

Figure 4. Natural frequencies and damping ratios of a cantilever beam using Waspmote wireless sensor node (MEMS accelerometer: LIS331DLH

The performance of low-cost wireless accelerometer is justified in terms of modal parameter identification. The accuracy of wireless vibration measurements in terms of natural frequencies is enough to replace the traditional wired accelerometer.

\section{V.CONCLUSION}

We justified the performance of low-resource wireless vibration measurement system by applying an experimental modal analysis. The dynamic properties of the structures such as natural frequencies, damping ratios, and mode shapes are obtained and compared to those of the conventional wired ICP vibration measurement system. The cantilevered steel beam is excited with the impact hammer. In this test, we used the fixed wireless accelerometer and the roving hammer as excitation and give a multiple-input, single-output (MISO) analysis. We have chosen 10 excitation points as an input force to obtain 3 mode shapes. The frequency response function is acquired by computation of the cross power spectrum of the input and output vibration data using commercial Smart Office Analyzer software. The results have shown that the low-cost, off-the-shelf wireless sensor nodes with MEMS accelerometer works very efficiently, enabling us to conclude that they should play an important part in forming wireless sensor networks for low-cost structural health monitoring

\section{ACKNOWLEDGMENTS}

This research was supported by Basic Science Research Program through the National Research Foundation of Korea(NRF) funded by the Ministry of Education (No. 2014-0024242).

\section{REFERENCES}

[1] Y. Wang, J.P. Lynch, and K.H. Law, "A wireless structural health monitoring system with multithreaded sensing devices: design and validation," Structure and Infrastructure Engineering, Vol. 3, No. 2, pp. 103-120, 2007.

[2] B.J. Schwarz and M.H. Richardson, "Experimental Modal Analysis," CSI Reliability Week, Orlando, FL 1999.

[3] P.D. Chougule, P.H. Kirkegaard and S.R.K. Nielsen, "Low Cost Wireless Sensor Network for Structural Health Monitoring," In Scandinavian Vibration Forum (SVIB), 2010.

[4] Waspmote Datasheet, http://www.libelium.com/products/waspmote

[5] Smart Office Analyzer, https://www.mpihome.com/en/products-solutions/dynamic-signal-analysis.html

[6] H.A. Al-Khazali and M. Askari, "Calculations of Frequency Response Functions Using Computer Smart Office Software and Nyquist Plot under Gyroscopic Effect Rotation," IRACST - Int'l J. of Computer Science and Information Technology \& Security, Vol. 1, No. 2, 2011.

[7] P. Avitabile, "Experimental modal analysis. Sound and vibration," Vol. 35, No. 1, pp. 21-31A, 2001. 